\title{
Clustering of metabolic syndrome components in a Middle Eastern diabetic and non-diabetic population
}

\author{
Alireza Esteghamati*1, Ali Zandieh¹, Omid Khalilzadeh¹, Alipasha Meysamie² and Haleh Ashraf3
}

\begin{abstract}
Background: Metabolic syndrome (MetS) encompasses a cluster of coronary heart disease and diabetes mellitus risk factors. In this study, we aimed to elucidate the factors underlying the clustering of MetS components in diabetic and non-diabetic individuals.

Methods: Factor analysis was performed on 2978 (1652 non-diabetic and 1326 diabetic) participants. Entering waist circumference, homeostasis model assessment of insulin resistance (HOMA-IR), triglycerides, high-density lipoproteincholesterol (HDL-C) and systolic blood pressure (SBP), we performed exploratory factor analysis in diabetic and nondiabetic individuals separately. The analysis was repeated after replacing triglycerides and HDL-C with triglycerides to $\mathrm{HDL}-\mathrm{C}$ ratio (triglycerides/HDL-C). MetS was defined by either adult treatment panel III (ATPIII), international diabetes federation (IDF) criteria, or by the modified form of IDF using waist circumference cut-off points for Iranian population.

Results: The selection of triglycerides and HDL-C as two distinct variables led to identifying two factors explaining $61.3 \%$ and $55.4 \%$ of the total variance in non-diabetic and diabetic participants, respectively. In both diabetic and nondiabetic subjects, waist circumference, HOMA-IR and SBP loaded on factor 1. Factor 2 was mainly determined by triglycerides and HDL-C. Factor 1 and 2 were directly and inversely associated with MetS, respectively. When triglycerides and $\mathrm{HDL}-\mathrm{C}$ were replaced by triglycerides/HDL-C, one factor was extracted, which explained $47.6 \%$ and $38.8 \%$ of the total variance in non-diabetic and diabetic participants, respectively.

Conclusion: This study confirms that in both diabetic and non-diabetic participants the concept of a single underlying factor representing MetS is plausible.
\end{abstract}

\section{Background}

Metabolic syndrome (MetS) encompasses a cluster of coronary heart disease and diabetes mellitus risk factors, including abdominal obesity, glucose intolerance, dyslipidemia and elevated blood pressure [1,2]. Subjects with MetS tend to have high insulin levels, reflecting greater insulin resistance [1-3]. Insulin resistance or hyperinsulinemia has been suggested to be the underlying characteristic of MetS, although a central role for insulin resistance is still controversial [3]. The understanding of how MetS components cluster together can help clinicians interpret the MetS pathophysiology and develop effective strategies for identifying and preventing the

* Correspondence: esteghamati@tums.ac.ir

1 Endocrinology and Metabolism Research Center (EMRC), Vali-Asr Hospital, School of Medicine, Tehran University of Medical Sciences, Tehran, Iran Full list of author information is available at the end of the article inherent risk of coronary heart disease and diabetes mellitus. The multitude of clinical and biochemical alterations resembling the MetS, the strong cross-linkage of involved pathways and multiple feedback mechanisms, complicate the identification of the events which lead to the cascade of disorders that characterize the syndrome [4]. Moreover, statistically strong intercorrelations among various features of MetS, also complicate establishing independent associations using standard multivariate statistical models [2,3]. Factor analysis is potentially a way of advancing this body of research by explaining the correlation between the components of the syndrome in terms of a small set of latent factors, providing insights into the underlying process. Exploratory factor analysis is a hypotheses generation method, in which the number of factors is essentially unknown and has to be determined 
from data. Extracting one factor implies that a single underlying pathophysiologic mechanism contributes to the appearance of the syndrome. If two or more factors were extracted, the existence of a unique syndrome would be called into question [3].

Previous studies have entered different numbers of variables ranged from 4 to 21 in factor analysis and extracted from 1 to 7 factors [2,5-13]. Some investigations have identified three or four factors, implying a possible heterogeneity of the MetS [2,7,9-11]. Since there appears to be ethnic differences in the expression of MetS [1], different findings may arise on the basis of sample collection. For example, hypertension may not be associated with MetS in American Indians and African Americans [14]. Variations in the extracted factors can also be result of discrepancies in numbers and nature of considered components. For example some studies include insulin levels while others do not; the same is true for uric acid, leptin and waist to hip ratio [2,5-13]. Since factor analysis extracts factors due to the interrelatedness of measured variables, using two or more measures for the same trait (e.g. systolic blood pressure [SBP] and diastolic blood pressure $[\mathrm{DBP}]$ ) leads to find more factors than expected [15]. These variables compared to other variables are highly associated with each other, thus tend to load on a separate factor. This phenomenon can also be observed with fasting and postprandial glucose or with high density lipoprotein cholesterol (HDL-C) and triglycerides (TG) $[5,16]$.

Factor analysis of the MetS has been the focus of little attention in the Middle Eastern ethnicity; furthermore, the clustering of MetS components is yet to be more explored in the diabetic patients. In this study, for the first time we performed factor analysis on the components of MetS in a large sample of Iranian diabetic and non-diabetic participants. MetS can be defined according to Adult Treatment Panel (ATPIII) [1] or International Diabetes Federation (IDF) criteria [17]. We recently proposed that the optimal waist circumference cut-off points for the diagnosis of IDF defined MetS in Iranian adults would be different from those of other populations [18]. In this study, we also explored the influence of the extracted factors on different definitions of MetS.

\section{Methods}

\section{Participants}

A total of 3023 participants (aged 18-75 yrs), who were referred consecutively from September 2005 to December 2008 to one of the four Tehran University-affiliated health service centers located in east, west, south and center of Tehran, were studied. Participants were enrolled from individuals taking health examinations, or those who accompanied patients. Subjects who needed special concern beyond routine examination for their symptoms (i.e. patients with chronic liver disease, renal, thyroid or adrenal problems) along with those under insulin therapy were not included in the study. Diabetic participants were taking metformin, glibenclamide or both simultaneously for controlling their hyperglycemia. Due to missing data, 45 participants were excluded, resulting in a total of 2978 subjects. Oral informed consent was obtained from all individuals before study commencement. The study was conducted in accordance with Helsinki declaration and was performed in line with considerations, recommended by local ethics review committee of Tehran University of Medical Sciences.

\section{Procedures}

Waist circumference was measured at mid-distance between iliac crest and rib cage and was rounded to the nearest $0.1 \mathrm{~cm}$. The participants were instructed to rest for at least $5 \mathrm{~min}$ before having their blood pressure checked twice with at least 5 min interval. Venous blood samples were collected following $12 \mathrm{~h}$ overnight fast. Fasting plasma glucose was measured by glucose-oxidase method. TG and HDL-C were determined by enzymatic methods (Parsazmun, Karaj, Iran). Insulin was measured by radioimmunoassay, using an antibody with no crossreaction against pro-insulin and C-peptide (Immunotech, Prague, Czech Republic). The intra and inter-assay coefficients of variation were lower than 4.3 and 3.4 , respectively.

\section{Definitions}

The homeostasis model assessment of insulin resistance (HOMA-IR) was calculated as fasting insulin $(\mathrm{U} / \mathrm{l}) \times$ fasting plasma glucose $(\mathrm{mg} / \mathrm{dl}) / 405$, as described by Matthews et al. [19]. Diabetes mellitus was diagnosed according to the criteria of American Diabetes Association [20]. The average of two obtained SBPs was used in this study. MetS was defined due to either original ATPIII or IDF declarations. ATPIII criteria allow the diagnosis of MetS when three or more of the following conditions are satisfied: presence of the abdominal obesity (waist circumference $\geq 102 \mathrm{~cm}$ and $\geq 88 \mathrm{~cm}$ in men and women, respectively), elevated blood pressure ( $\mathrm{SBP} \geq 130 \mathrm{mmHg}$ and/or DBP $\geq 85 \mathrm{mmHg}$ ), low HDL-C $(<40 \mathrm{mg} / \mathrm{dl}$ and $<50$ $\mathrm{mg} / \mathrm{dl}$ in men and women, respectively), TG $\geq 150 \mathrm{mg} / \mathrm{dl}$ and fasting plasma glucose $\geq 100 \mathrm{mg} / \mathrm{dl}$ (or diabetes) $[1,21]$. According to original IDF criteria a person with MetS must have abdominal obesity (waist circumference $\geq 94 \mathrm{~cm}$ and $\geq 80 \mathrm{~cm}$ in men and women, respectively) plus any two or more of the following conditions: elevated blood pressure (see above) or treatment of previously diagnosed hypertension, low HDL-C (see above) or on HDL-C therapy, TG $\geq 150 \mathrm{mg} / \mathrm{dl}$ or on TG therapy and fasting plasma glucose $\geq 100 \mathrm{mg} / \mathrm{dl}$ (or diabetes) [17]. MetS was also defined by the modified form of IDF crite- 
ria in which the waist circumference cut-offs were substituted by optimal values (waist circumference $\geq 90 \mathrm{~cm}$ in both men and women) for use in Iranian population [18].

\section{Statistical analysis}

Data were analyzed using SPSS software (version 16.0; SPSS Inc., Chicago, USA). To extrapolate our results to the population of Tehran we carried out a complex sample survey analysis. The data were directly weighted for age (10-year strata) and sex distribution of diabetic and non-diabetic residents of Tehran, according to the results of an epidemiological study on patterns of diabetes, conducted by the Ministry of Health [22], and the data of the national censuses of Iran in 2006. To compare the principal characteristics of diabetic and non-diabetic participants, we used the method of general linear modeling in complex sample analysis mode using the F statistic. Comparisons for categorical variables were made by designbased $x^{2}$ analysis. The relationship between individual variables was examined using bivariate Pearson's correlation coefficients. Since a large number of analyses were carried out, a $P<0.01$ was considered statistically significant. In order to improve the normality of skewed variables (i.e. HOMA-IR, TG and TG to HDL-C ratio) natural $\log$ transformations were used in the subsequent analyses as appropriate. Factor analysis was performed in two separate models. In model 1 waist circumference, SBP, HOMA-IR, TG and HDL-C were selected for analyses. Exploratory factor analysis was performed using principal component analysis, a technique for reducing the number of original variables into fewer latent factors. The variables cluster on the basis of linear correlations that exist among them. Factors with an eigenvalue (the amount of variance attributable to the factor) of greater than one were extracted and transformed by varimax rotation method to enhance interpretation. Varimax method maintains the independence of extracted factors and simplifies the interpretation of analysis by maximizing factor loadings to one or minimizing it to zero. Varimax rotation cannot be performed if factor analysis extracts just one factor. Factor scores, which are estimates of individual factors with the mean and standard deviation equal to 0 and 1 , respectively, were determined by regression method. The factor loading of a variable on a factor equals the correlation coefficient between that variable and the factor score. In accordance with several previous reports, factor loadings of $\geq|0.40|$ were considered meaningful for interpretation; because this ensures that the variable shares at least $15 \%$ of the variance with the factor $[2,6,8,9,11-13,15]$. Factor analysis was performed in diabetic and non-diabetic participants separately. Binary logistic regression analysis was used to assess the association of factor scores with MetS defined by either ATPIII or original IDF or by the modified form of IDF criteria.

Considering that two of the variables entered in analyses are related to lipid profile (i.e. TG and HDL-C), in model 2 we repeated all aforementioned analyses after substituting ratio of TG to HDL-C for both TG and HDLC.

\section{Results}

\section{Descriptive statistics}

Sex-specific principal characteristics of our study population in diabetic and non-diabetic participants are presented in Table 1 . In men the mean ages \pm SEM of diabetic and non-diabetic participants were $53.8 \pm 0.5$ and $41.1 \pm 0.7$ years and in women were $54.1 \pm 0.4$ and $40.0 \pm 0.4$ years, respectively. There were significant differences between all variables mentioned in Table 1, except insulin concentration. After adjusting for age and sex, in diabetic participants the prevalence of MetS defined by ATPIII, original IDF and modified IDF criteria (74\%, $76 \%$ and $69 \%$, respectively) was higher than nondiabetic participants $(33 \%, 37 \%$ and $32 \%$, respectively, $P$ $<0.001$ )

\section{Univariate associations}

In non-diabetic participants, all variables entered in analysis were correlated with each other except HDL-C and SBP. However, in diabetic participants, SBP was merely associated with waist circumference (Table 2).

\section{Exploratory factor analysis and logistic regression}

Entering waist circumference, HOMA-IR, TG, HDL-C and SBP as variables (components of MetS) in model 1, exploratory factor analysis extracted two factors in both non-diabetic and diabetic participants (Table 3 and Figure 1). Generally, factor 1 was related to waist circumference, HOMA-IR and SBP while factor 2 was mainly determined by TG and HDL-C (Table 3). In non-diabetic participants TG also loaded positively on factor 1 . HDL$\mathrm{C}$ loaded positively and TG negatively on factor 2 . These two factors together explained $61.3 \%$ and $55.4 \%$ of the total variance in non-diabetic and diabetic participants, respectively.

In model 2, substituting TG and HDL-C with TG to HDL-C ratio led to a single factor structure for both nondiabetic and diabetic participants, which explained $47.6 \%$ and $38.8 \%$ of variance, respectively (Table 3). HOMA-IR, waist circumference and TG to HDL-C ratio had large factor loadings on the selected factor of model 2 in both diabetic and non-diabetic participants. However, in diabetic participants SBP had lower factor loading than did other variables and thus was less correlated with the factor (Table 3). 
Table 1: Principal Characteristics of the Participants $(n=2978)$.

\begin{tabular}{|c|c|c|c|c|}
\hline \multirow[t]{2}{*}{ Characteristic } & \multicolumn{2}{|c|}{ Men } & \multicolumn{2}{|c|}{ Women } \\
\hline & $\begin{array}{l}\text { Non-diabetic participants } \\
(n=397)\end{array}$ & $\begin{array}{l}\text { Diabetic participants } \\
(n=565)\end{array}$ & $\begin{array}{l}\text { Non-diabetic participants } \\
(n=1255)\end{array}$ & $\begin{array}{l}\text { Diabetic participants } \\
(n=761)\end{array}$ \\
\hline Age (years) & $41.1 \pm 0.7$ & $53.8 \pm 0.5^{*}$ & $40.0 \pm 0.4$ & $54.1 \pm 0.4^{*}$ \\
\hline Waist circumference (cm) & $93.2 \pm 0.6$ & $99.5 \pm 0.4^{*}$ & $90.9 \pm 0.4$ & $97.9 \pm 0.4^{*}$ \\
\hline Fasting plasma glucose (mg/dl) & $90.1 \pm 0.5$ & $174.3 \pm 2.5^{*}$ & $91.5 \pm 0.3$ & $171.8 \pm 2.1^{*}$ \\
\hline Insulin (U/I) & $8.1 \pm 0.3$ & $8.9 \pm 0.2$ & $9.2 \pm 0.2$ & $9.0 \pm 0.2$ \\
\hline HOMA-IR (units)a & $1.8 \pm 0.08$ & $3.7 \pm 0.11^{*}$ & $2.1 \pm 0.04$ & $3.7 \pm 0.09^{*}$ \\
\hline TG (mg/dl) & $162.2 \pm 4.7$ & $208.7 \pm 6.8^{*}$ & $133.7 \pm 2.1$ & $198.8 \pm 4.1^{*}$ \\
\hline HDL-C (mg/dl) & $46.8 \pm 0.6$ & $42.1 \pm 0.5^{*}$ & $50.9 \pm 0.3$ & $48.5 \pm 0.4^{*}$ \\
\hline $\mathrm{SBP}(\mathrm{mmHg})$ & $121.1 \pm 0.8$ & $127.8 \pm 0.7^{*}$ & $115.2 \pm 0.5$ & $131.9 \pm 0.7^{*}$ \\
\hline \multicolumn{5}{|l|}{ Abdominal obesity $(\%)^{\mathrm{b}}$} \\
\hline ATP III & 22.7 & $36.0^{*}$ & 54.0 & $80.7^{*}$ \\
\hline IDF & 48.5 & $72.0^{*}$ & 78.6 & $95.8^{*}$ \\
\hline Modified IDF & 58.8 & $85.7^{*}$ & 51.2 & $76.9^{*}$ \\
\hline $\mathrm{TG} \geq 150(\%)$ & 43.3 & $59.9 *$ & 33.7 & $65.8^{*}$ \\
\hline Low HDL-C (\%)c & 26.8 & $46.4^{*}$ & 46.0 & $57.6^{*}$ \\
\hline Elevated blood pressure (\%)d & 35.6 & $56.8^{*}$ & 27.4 & $65.7^{*}$ \\
\hline \multicolumn{5}{|l|}{ MetS (\%)e } \\
\hline ATP III & 23.0 & $67.6^{*}$ & 31.3 & $87.7^{*}$ \\
\hline IDF & 28.1 & $66.1^{*}$ & 36.0 & $89.9^{*}$ \\
\hline Modified IDF & 31.2 & $78.1^{*}$ & 26.8 & $72.9^{*}$ \\
\hline
\end{tabular}

Variables are expressed as percentage or mean \pm SEM

* $P<0.001$ for the comparison of diabetic and non-diabetic participants. a Fasting insulin $(\mathrm{U} / \mathrm{l}) \times$ fasting plasma glucose $(\mathrm{mg} / \mathrm{dl}) / 405$. b, c, dand e See text for definitions.

Abbreviations: HOMA-IR, homeostasis model assessment of insulin resistance; TG, triglycerides; HDL-C, high density lipoprotein cholesterol; SBP, systolic blood pressure; MetS, metabolic syndrome.

Table 4 shows the association of factor scores with MetS, defined by conventional criteria. In model 1 , in both non-diabetic and diabetic participants, factor 1 was directly associated with MetS (odds ratio $[\mathrm{OR}]>1$ ). Nevertheless, factor 2 and MetS were inversely correlated with each other $(\mathrm{OR}<1)$. In model 2 , the ORs $(95 \%$ confidence interval) for MetS defined by ATPIII, original IDF and modified IDF were 9.9 (7.8-12.6), 8.4 (6.8-10.4) and 12.4 $(9.5-16.0)$ in non-diabetic subjects and $5.8(4.6-7.3), 4.4$ (3.6-5.4) and 7.4 (5.8-9.4) in diabetic subjects, respectively.

\section{Discussion}

Factor analysis of components revealed two factors in model 1. In diabetic participants the first factor was related to waist circumference, HOMA-IR and SBP while in non-diabetic participants it was also associated with TG. The second factor was determined positively by HDL-C and negatively by TG. Factor 1 and 2 were directly and inversely associated with MetS, respectively.
When TG and HDL-C were replaced by a composite measure, which included both of them (i.e. TG to HDL-C ratio), one factor was found, confirming previous observations on the monofactorial structure of MetS [23]. Furthermore, these findings are consistent with the concept that using two or more measures for the same trait results in an overfactored model.

As previously mentioned, variations in sample collection, number and nature of selected components and also in methods used for interpretation of analysis preclude formal direct comparisons, but an overview elucidates some common patterns. In several previous studies insulin resistance loaded on the same factor with measures of obesity including weight, body mass index and waist circumference $[2,5-9,11,13]$. Furthermore, measures of obesity were more likely to belong to the first extracted factor and to have the highest factor loadings [6-9, 13]. It has been demonstrated that visceral fat via its tendency to release free fatty acids, stimulates hepatic glucose production and accentuates insulin resistance [24]. More- 
Table 2: Correlation coefficients between variables associated with metabolic syndrome in non-diabetic $(n=1652)$ and diabetic $(n=1326)$ participants.

\begin{tabular}{|c|c|c|c|c|c|}
\hline Variable & Waist circumference & HOMA-IRa & TG & HDL-C & TG/HDL-C \\
\hline \multicolumn{6}{|c|}{ Non-diabetic participants } \\
\hline HOMA-IRa, b & $0.44^{* *}$ & & & & \\
\hline TGb & $0.35^{* *}$ & $0.27^{* *}$ & & & \\
\hline HDL-C & $-0.13^{* *}$ & $-0.14^{* *}$ & $-0.28^{* *}$ & & \\
\hline $\mathrm{TG} / \mathrm{HDL}-\mathrm{C}^{\mathrm{b}}$ & $0.34^{* *}$ & $0.28^{* *}$ & $0.93^{* *}$ & $-0.61^{* *}$ & \\
\hline SBP & $0.37^{* *}$ & $0.11^{* *}$ & $0.27^{* *}$ & 0.01 & $0.22^{* *}$ \\
\hline \multicolumn{6}{|c|}{ Diabetic participants } \\
\hline HOMA-IRa, b & $0.36^{* *}$ & & & & \\
\hline TGb & $0.18^{* *}$ & $0.25^{* *}$ & & & \\
\hline $\mathrm{HDL}-\mathrm{C}$ & $-0.08^{*}$ & $-0.11^{* *}$ & $-0.31^{* *}$ & & \\
\hline $\mathrm{TG} / \mathrm{HDL}-\mathrm{C}^{\mathrm{b}}$ & $0.18^{* *}$ & $0.24^{* *}$ & $0.93^{* *}$ & $-0.63^{* *}$ & \\
\hline SBP & $0.09^{*}$ & 0.05 & 0.03 & 0.05 & 0.01 \\
\hline
\end{tabular}

${ }^{*} P<0.01 ;{ }^{*} P<0.001$. a Fasting insulin $(\mathrm{U} / \mathrm{l}) \times$ fasting glucose $(\mathrm{mg} / \mathrm{dl}) / 405$. ${ }^{\text {b }}$ Log transformed.

Abbreviations: HOMA-IR, homeostasis model assessment of insulin resistance; TG, triglycerides; HDL-C, high density lipoprotein cholesterol; TG/HDL-C, triglycerides to HDL-C ratio; SBP, systolic blood pressure.

over, visceral fat cells release some agents to the blood (e.g. plasminogen activator inhibitor-1, interleukin-6 and tumor necrosis factor- $\alpha$ ) that are possibly linked to some aspects of insulin resistance [24]. These interrelated pathophysiologic mechanisms, underlying the association of insulin resistance and obesity, may play a principal role in the expression of MetS and its complications including coronary heart disease and diabetes.

MetS is believed to be related to insulin resistance which can be scored by measuring fasting insulin concentration [1-3]. However, due to the fact that in diabetic patients increase in blood glucose and insulin resistance is accompanied by impaired insulin secretion [4], and since there was no significant difference in fasting insulin concentration between diabetic and non-diabetic participants (Table 1); fasting insulin is not a reliable index of insulin resistance in diabetic patients. It has been demonstrated that there is a good correlation between estimates of insulin resistance derived from HOMA-IR and from the euglycemic clamp [25]. Moreover HOMA-IR is a reliable index in diabetic patients and has been widely used as an estimate of insulin resistance in both non-diabetic and diabetic subjects $[25,26]$. Besides these advantages, HOMA-IR has also some limitations. For instance, in Korean population, the validity of HOMA-IR as a surrogate measure of insulin resistance in lean type 2 diabetic subjects with the insulin secretory defect is disputed [27].
In model 1 of our analyses, HOMA-IR loaded primarily on factor one, but the factor loading of HOMA-IR on factor 2 was -0.35 in non-diabetic participants which is close to the loading threshold, $|0.40|$. Further, TG was associated with both extracted factors in non-diabetic participants (Figure 1). Many studies have used lower loading thresholds or noted the effect of relaxing the threshold on the findings $[6,7,12,15]$. Even Cureton and D'Agostino suggested a loading threshold as low as 0.2 for factor interpretation [28]. In model 1, association of HOMA-IR and TG with both extracted factors in non-diabetic participants shows that the two factors are related to each other and are not totally independent. Consistent with our findings, in most studies measures of insulin resistance load on more than one factor, suggesting insulin resistance as a unifying theme underlying the MetS [3]. On the other hand, Shen et al. found that TG and HDL-C are part of the same risk component for MetS and inclusion of both may be redundant [29]. In this regard, TG to HDL-C ratio is reported to be helpful in determining risk of coronary heart disease [30]. In model 2, substitution of TG and HDL-C with TG to HDL-C ratio led to extraction of just one factor. Mannucci et al. reported similar results and revealed that considering TG and HDL-C as two different components of MetS results in overestimation of the role of dyslipidaemia in MetS [5]. These findings suggest that the model 2 of our analyses (one-factor model) 
Table 3: Factor loadings of variables on the selected factors of Model 1 and 2, in diabetic and non-diabetic participants.

\begin{tabular}{|c|c|c|c|c|}
\hline \multirow{2}{*}{\begin{tabular}{|c|} 
Variable \\
Model 1a
\end{tabular}} & \multicolumn{2}{|c|}{$\begin{array}{l}\text { Non-diabetic participants } \\
(n=1652)\end{array}$} & \multicolumn{2}{|c|}{$\begin{array}{l}\text { Diabetic participants } \\
(n=1326)\end{array}$} \\
\hline & Factor 1 & Factor 2 & Factor 1 & Factor 2 \\
\hline Waist circumference & $0.80^{*}$ & -0.14 & $0.76^{*}$ & -0.12 \\
\hline HOMA-IR & $0.56^{*}$ & -0.35 & $0.71^{*}$ & -0.25 \\
\hline TGb & $0.57^{*}$ & $-0.46^{*}$ & 0.31 & $-0.69^{*}$ \\
\hline $\mathrm{HDL}-\mathrm{C}$ & 0.00 & $0.89^{*}$ & 0.03 & $0.81^{*}$ \\
\hline SBP & $0.75^{*}$ & 0.29 & $0.50^{*}$ & 0.36 \\
\hline$\%$ Total variance explained & 36.8 & 24.5 & 28.6 & 26.8 \\
\hline Model 2 & \multicolumn{2}{|l|}{ Factor 1} & \multicolumn{2}{|l|}{ Factor 1} \\
\hline Waist circumference & \multicolumn{2}{|l|}{$0.82^{*}$} & \multicolumn{2}{|l|}{$0.74^{*}$} \\
\hline HOMA-IR & \multicolumn{2}{|l|}{$0.68^{*}$} & \multicolumn{2}{|l|}{$0.77^{*}$} \\
\hline $\mathrm{TG} / \mathrm{HDL}-\mathrm{C}^{\mathrm{b}}$ & \multicolumn{2}{|l|}{$0.66^{*}$} & \multicolumn{2}{|l|}{$0.59 *$} \\
\hline SBP & \multicolumn{2}{|l|}{$0.58^{*}$} & \multicolumn{2}{|l|}{0.21} \\
\hline$\%$ total variance explained & \multicolumn{2}{|l|}{47.6} & \multicolumn{2}{|l|}{38.8} \\
\hline
\end{tabular}

Factors with an eigenvalue $\geq 1$ were selected for analysis. ${ }^{*}$ Factor loadings $\geq|0.40|$. a Factor loadings are calculated after varimax rotation of variables in each extracted factor. ${ }^{b}$ Log transformed.

Abbreviations: HOMA-IR, homeostasis model assessment of insulin resistance; TG, triglycerides; HDL-C, high density lipoprotein cholesterol; TG/HDL-C, triglycerides to HDL-C ratio; SBP, systolic blood pressure.

is also plausible and support previous studies suggesting one underlying factor (perhaps insulin resistance) for the syndrome [23], the point which is recently substantiated in experimental studies [31].

Insulin resistance and hyperinsulinemia lead to hypertension via a number of mechanisms, including renal sodium and water retention, rennin-angiotensin-aldosterone system, plasma noradrenaline and sympathetic nervous system activity [32,33]. Since subjects' age range is wide (18-75 years) it is more conceivable to use just SBP instead of mean arterial pressure [34]. In model 2, the factor loading of SBP was lowest among components of MetS implying it as the most tenuously linked component. Review of previous studies shows that in most analyses measures of blood pressure load on a unique and separate factor, which does not overlap with other factors

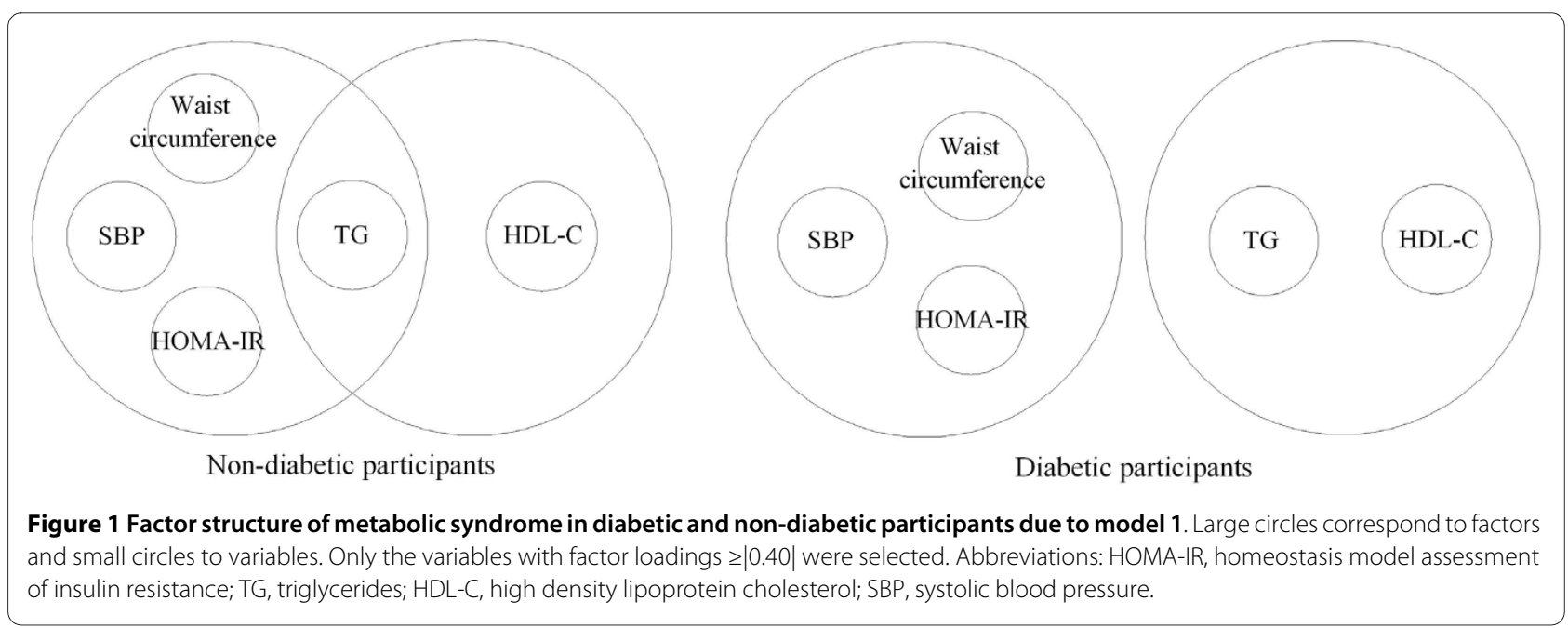


Table 4: Odds ratios (95\% confidence interval) of factor scores for metabolic syndrome in binary logistic regression in model 1 and 2, Tehran, Iran

\begin{tabular}{|c|c|c|c|c|}
\hline Criteria & Non-diabetic ( $n$ & & Diabetic $(n=$ & \\
\hline Model 1 & Factor 1 & Factor 2 & Factor 1 & Factor 2 \\
\hline ATP III & $8.9(7.0-11.2)$ & $0.37(0.32-0.44)$ & $5.0(0.4-6.3)$ & $0.38(0.31-0.46)$ \\
\hline IDF & $7.4(6.0-9.1)$ & $0.34(0.29-0.40)$ & $4.7(3.8-5.8)$ & $0.66(0.56-0.78)$ \\
\hline modified IDF & $10.6(8.3-13.7)$ & $0.35(0.29-0.41)$ & $7.0(5.5-8.9)$ & $0.52(0.43-0.62)$ \\
\hline Model 2 & Factor 1 & & Factor 1 & \\
\hline ATP III & $9.9(7.8-12.6)$ & & $5.8(4.6-7.3)$ & \\
\hline IDF & $8.4(6.8-10.4)$ & & $4.4(3.6-5.4)$ & \\
\hline Modified IDF & $12.4(9.5-16.0)$ & & $7.4(5.8-9.4)$ & \\
\hline
\end{tabular}

of MetS [2,9,11-13], revealing weak relation between blood pressure and other components of MetS [3,29]. Even, such relation may not be evident in some ethnic groups like American Indians and African Americans [14]. Moreover, we found that SBP is less correlated with components of MetS especially in diabetic subjects (Table 2 ). These findings suggest that blood pressure is less influenced by the common pathophysiologic pathway which underlies the MetS.

Ethnic differences in the expression of MetS are linked to different appropriate waist circumference cut-offs for determination of abdominal obesity. It has been found that Asians in comparison to Caucasians are more prone to obesity related comorbidities even at lower waist circumference values [35]. We recently showed that waist circumference cut-offs recommended by IDF are not appropriate in Iranian population and should change to $90 \mathrm{~cm}$ in both men and women [18]. In this study we found that IDF definition modified by our recommended waist circumference cut-offs is strongly associated with extracted factors. As table 4 shows, factor 2 was inversely associated with MetS and therefore had protective roles against it, which seems reasonable because factor 2 was mainly characterized by significant positive factor loading for HDL-C.

The major limitation, relevant to our results interpretation, is use of cross-sectional data. However, consistent with our analysis most previous studies have used crosssectional study design to evaluate the interrelatedness of MetS components. Cross-sectional analyses provides information at a single point in time, nevertheless progressive and reciprocal mechanisms are likely to be involved in the appearance of MetS. Therefore, longitudi- nal studies are suggested to improve better our understanding of MetS.

\section{Conclusions}

This study revealed that in both diabetic and non-diabetic individuals the concept of a single underlying factor (pathophysiologic pathway) for the expression of MetS is plausible. Further, we showed that similar to ATPIII and original IDF definitions; modified IDF definition is also strongly associated with identified factors. Nevertheless, among components of MetS blood pressure is less influenced by the common factor and could be also mediated by other pathophysiologic processes.

\section{Abbreviations}

MetS: metabolic syndrome; SBP: systolic blood pressure; DBP: diastolic blood pressure; HDL-C: high density lipoprotein cholesterol; TG: triglycerides; ATPIII: adult treatment panel III; IDF: international diabetes federation; HOMA-IR: homeostasis model assessment of insulin resistance; OR: odds ratio.

\section{Competing interests}

The authors declare that they have no conflict of interests.

\section{Authors' contributions}

$\mathrm{AE}$ conceived the study, participated in its design, coordination and acquisition of data. AZ wrote the manuscript and performed the statistical analysis and participated in the acquisition of data. OK helped to draft the manuscript and interpret the data and participated in the acquisition of data. AM helped to perform the statistical analysis. HA helped to draft the manuscript and participated in the acquisition of data. All authors read and approved the final manuscript.

\section{Author Details}

1 Endocrinology and Metabolism Research Center (EMRC), Vali-Asr Hospital, School of Medicine, Tehran University of Medical Sciences, Tehran, Iran, 2Department of Preventive Medicine, School of Medicine, Tehran University of Medical Sciences, Tehran, Iran and 'BDepartment of Cardiology, Tehran Heart Center, Tehran University of Medical Sciences, Tehran, Iran 
Received: 17 December 2009 Accepted: 8 June 2010

Published: 8 June 2010

\section{References}

1. Grundy SM, Hansen B, Smith SC, Cleeman JI, Kahn RA: Clinical management of metabolic syndrome: report of the American Heart Association/National Heart, Lung, and Blood Institute/American Diabetes Association conference on scientific issues related to management. Circulation 2004, 109(4):551-556.

2. Hanson RL, Imperatore G, Bennett PH, Knowler WC: Components of the "metabolic syndrome" and incidence of type 2 diabetes. Diabetes 2002, 51(10):3120-3127.

3. Meigs JB: Invited commentary: insulin resistance syndrome? Syndrome $\mathrm{X}$ ? Multiple metabolic syndrome? A syndrome at all? Factor analysis reveals patterns in the fabric of correlated metabolic risk factors. Am J Epidemiol 2000, 152(10):908-911.

4. Ferrannini E: Insulin resistance versus insulin deficiency in non-insulindependent diabetes mellitus: problems and prospects. Endocr Rev 1998, 19(4):477-490.

5. Mannucci E, Monami M, Rotella CM: How many components for the metabolic syndrome? Results of exploratory factor analysis in the FIBAR study. Nutr Metab Cardiovasc Dis 2007, 17(10):719-726.

6. Pyorala M, Miettinen $\mathrm{H}$, Halonen $\mathrm{P}$, Laakso M, Pyorala K: Insulin resistance syndrome predicts the risk of coronary heart disease and stroke in healthy middle-aged men: the 22-year follow-up results of the Helsinki Policemen Study. Arterioscler Thromb Vasc Biol 2000, 20(2):538-544.

7. Lakka HM, Laaksonen DE, Lakka TA, Niskanen LK, Kumpusalo E, Tuomilehto J, Salonen JT: The metabolic syndrome and total and cardiovascular disease mortality in middle-aged men. JAMA 2002, 288(21):2709-2716.

8. Hodge AM, Boyko EJ, de Courten M, Zimmet PZ, Chitson P, Tuomilehto J, Alberti KG: Leptin and other components of the Metabolic Syndrome in Mauritius--a factor analysis. Int $J$ Obes Relat Metab Disord 2001, 25(1):126-131

9. Hanley AJ, Festa A, D'Agostino RB, Wagenknecht LE, Savage PJ, Tracy RP, Saad MF, Haffner SM: Metabolic and inflammation variable clusters and prediction of type 2 diabetes: factor analysis using directly measured insulin sensitivity. Diabetes 2004, 53(7):1773-1781.

10. Oh JY, Hong YS, Sung YA, Barrett-Connor E: Prevalence and factor analysis of metabolic syndrome in an urban Korean population. Diabetes Care 2004, 27(8):2027-2032.

11. Austin MA, Edwards KL, McNeely MJ, Chandler WL, Leonetti DL, Talmud PJ, Humphries SE, Fujimoto WY: Heritability of multivariate factors of the metabolic syndrome in nondiabetic Japanese americans. Diabetes 2004, 53(4):1166-1169.

12. Lafortuna CL, Adorni F, Agosti F, Sartorio A: Factor analysis of metabolic syndrome components in obese women. Nutr Metab Cardiovasc Dis 2008, 18(3):233-241.

13. Sakkinen PA, Wahl P, Cushman M, Lewis MR, Tracy RP: Clustering of procoagulation, inflammation, and fibrinolysis variables with metabolic factors in insulin resistance syndrome. Am J Epidemiol 2000, 152(10):897-907.

14. Saad MF, Lillioja S, Nyomba BL, Castillo C, Ferraro R, De Gregorio M, Ravussin E, Knowler WC, Bennett PH, Howard BV, et al: Racial differences in the relation between blood pressure and insulin resistance. $\mathrm{NEng/J}$ Med 1991, 324(11):733-739.

15. Lawlor DA, Ebrahim S, May M, Davey Smith G: (Mis)use of factor analysis in the study of insulin resistance syndrome. Am J Epidemiol 2004, 159(11):1013-1018.

16. Salmenniemi U, Ruotsalainen E, Pihlajamaki J, Vauhkonen I, Kainulainen S, Punnonen K, Vanninen E, Laakso M: Multiple abnormalities in glucose and energy metabolism and coordinated changes in levels of adiponectin, cytokines, and adhesion molecules in subjects with metabolic syndrome. Circulation 2004, 110(25):3842-3848.

17. International Diabetes Federation. The IDF consensus worldwide definition of the metabolic syndrome [article online] 2006 [http:// www.idf.org/webdata/docs/IDF Meta def final.pdfl. Accessed August 10,2009

18. Esteghamati A, Abbasi M, Rashidi A, Meysamie A, Khalilzadeh O, Haghazali M, Asgari F, Nakhjavani M: Optimal waist circumference cut-offs for the diagnosis of metabolic syndrome in Iranian adults: results of the third national survey of risk factors of non-communicable diseases (SuRFNCD-2007). Diabet Med 2009, 26(7):745-746.

19. Matthews DR, Hosker JP, Rudenski AS, Naylor BA, Treacher DF, Turner RC Homeostasis model assessment: insulin resistance and beta-cell function from fasting plasma glucose and insulin concentrations in man. Diabetologia 1985, 28(7):412-419.

20. American Diabetes Association: Diagnosis and classification of diabetes mellitus. Diabetes Care 2008, 31(Suppl 1):S55-60

21. Genuth S, Alberti KG, Bennett P, Buse J, Defronzo R, Kahn R, Kitzmiller J, Knowler WC, Lebovitz H, Lernmark A, et al:: Follow-up report on the diagnosis of diabetes mellitus. Diabetes Care 2003, 26(11):3160-3167.

22. Ministry of Health Programs Reports: Prevalence of diabetes and impaired fasting glucose in the adult population of Iran: the First National Survey of Risk Factors for Non-Communicable Diseases of Iran. Ministry of Health and Medical Education, Tehran, Iran; 2005

23. Pladevall M, Singal B, Williams LK, Brotons C, Guyer H, Sadurni J, Falces C, Serrano-Rios M, Gabriel R, Shaw JE, et al: A single factor underlies the metabolic syndrome: a confirmatory factor analysis. Diabetes Care 2006, 29(1):113-122

24. Frayn KN: Visceral fat and insulin resistance--causative or correlative? $\mathrm{Br}$ J Nutr 2000, 83(Suppl 1):S71-77.

25. Wallace TM, Levy JC, Matthews DR: Use and abuse of HOMA modeling Diabetes Care 2004, 27(6):1487-1495

26. Katsuki A, Sumida Y, Gabazza EC, Murashima S, Furuta M, Araki-Sasaki R, Hori $Y$, Yano $Y$, Adachi $Y$ : Homeostasis model assessment is a reliable indicator of insulin resistance during follow-up of patients with type 2 diabetes. Diabetes Care 2001, 24(2):362-365.

27. Kang ES, Yun YS, Park SW, Kim HJ, Ahn CW, Song YD, Cha BS, Lim SK, Kim KR, Lee HC: Limitation of the validity of the homeostasis model assessment as an index of insulin resistance in Korea. Metabolism 2005, 54(2):206-211.

28. Cureton EE, D'Agostino RB: Factor analysis: an applied approach. Hillsdale, NJ: Lawrence Erlbaum Associates; 1983.

29. Shen BJ, Todaro JF, Niaura R, McCaffery JM, Zhang J, Spiro A, Ward KD: Are metabolic risk factors one unified syndrome? Modeling the structure of the metabolic syndrome X. Am J Epidemiol 2003, 157(8):701-711.

30. Bertoluci MC, Quadros AS, Sarmento-Leite R, Schaan BD: Insulin resistance and triglyceride/HDLc index are associated with coronary artery disease. Diabetol Metab Syndr 2010, 2:11.

31. Haas JT, Biddinger SB: Dissecting the role of insulin resistance in the metabolic syndrome. Curr Opin Lipido/ 2009, 20(3):206-210.

32. Hayashi T, Takai S, Yamashita C: Impact of the Renin-AngiotensinAldosterone-System on Cardiovascular and Renal Complications in Diabetes Mellitus. Curr Vasc Pharmacol 2010:1

33. Grassi G, Arenare F, Quarti-Trevano F, Seravalle G, Mancia G: Heart rate, sympathetic cardiovascular influences, and the metabolic syndrome. Prog Cardiovasc Dis 2009, 52(1):31-37.

34. Sesso HD, Stampfer MJ, Rosner B, Hennekens CH, Gaziano JM, Manson JE, Glynn RJ: Systolic and diastolic blood pressure, pulse pressure, and mean arterial pressure as predictors of cardiovascular disease risk in Men. Hypertension 2000, 36(5):801-807.

35. Snehalatha C, Viswanathan V, Ramachandran A: Cutoff values for normal anthropometric variables in asian Indian adults. Diabetes Care 2003, 26(5):1380-1384.

doi: $10.1186 / 1758-5996-2-36$

Cite this article as: Esteghamati et al., Clustering of metabolic syndrome components in a Middle Eastern diabetic and non-diabetic population Diabetology \& Metabolic Syndrome 2010, 2:36 